\title{
Auditory Modulation of Multisensory Representations
}

\author{
Alfred O. Effenberg, Tong-Hun Hwang, Shashank Ghai \& Gerd Schmitz \\ Leibniz University Hannover, 30167 Hannover, Germany \\ effenberg@sportwiss.uni-hannover.de
}

\begin{abstract}
Motor control and motor learning as well as interpersonal coordination are based on motor perception and emergent perceptuomotor representations. At least in early stages motor learning and interpersonal coordination are emerging heavily on visual information in terms of observing others and transforming the information into internal representations to guide owns behavior. With progressing learning, also other perceptual modalities are added when a new motor pattern is established by repeated physical exercises. In contrast to the vast majority of publications on motor learning and interpersonal coordination referring to a certain perceptual modality here we look at the perceptual system as a unitary system coordinating and unifying the information of all involved perceptual modalities. The relation between perceptual streams of different modalities, the intermodal processing and multisensory integration of information as a basis for motor control and learning will be the main focus of this contribution.

Multi-intermodal processing of perceptual streams results in multimodal representations and opens up new approaches to support motor learning and interpersonal coordination: Creating an additional perceptual stream adequately auditory movement information can be generated suitable to be integrated with information of other modalities and thereby modulating the resulting perceptuomotor representations without the need of attention and higher cognition. Here, the concept of a movement defined real-time acoustics is used to serve the auditory system in terms of an additional movement-auditory stream. Before the computational approach of kinematic real-time sonification is finally described, a special focus is directed to the level of adaptation modules of the internal models. Furthermore, this concept is compared with different approaches of additional acoustic movement information. Moreover, a perspective of this approach is given in a broad spectrum of new applications of supporting motor control and learning in sports and motor rehabilitation as well as a broad spectrum of joint action and interpersonal coordination between humans but also concerning human-robotinteraction.
\end{abstract}

Keywords: Interpersonal Coordination, Motor Control, Motor Learning, Movement Sonification, Multimodal Integration, Multimodal Perception, Perceptuomotor Representation 


\section{Introduction \\ The Emergence of Perceptual-motor Representations}

From different fields of research on motor behavior there is strong evidence for a comprehensive and direct impact of current perception on motor processes: Motor control is driven by perceptual information. Disturbances between perceptual information of different modalities result in earnest perturbations as demonstrated already by Kohler (1951, 1963) with prism inversion goggles. Adaption usually only takes place when a person is physically acting and is pointing out the relevance of an intermodal adjustment of simultaneous congruent perceptual streams which are configured by and allocated to the specific current motor activity. The action specific activation of efferent motor patterns triggered by the anticipation of related perceptual effects builds the key frame of the 'internal model'-theory, which has been adapted to the acquisition of motor behavior by Wolpert, Ghahramani and Jordan (1995). Motor learning, resulting in long lasting changes of motor behavior, is based on the emergence of new internal models or appropriate changes of already existing ones in terms of adaptations e.g.: Changes of the inverse model as well as the forward model or the couplings of both (Wolpert, Diedrichsen \& Flanagan 2011). All three references (motor control, adaptation, motor learning) verify the importance of the anticipation of specific perceptual effects, the activation of adequate motor patterns and the emergence of according perceptual streams with close temporal relations. The structural interrelation between these patterns is a key element of motor learning, motor control and adaptation and it highlights the essential relevance of related sensory/perceptual effects - specified by the executed action pattern and configuring bottom-up the perceptual streams. The figures $1 \mathrm{a} \& \mathrm{~b}$ below illustrate the concept of a modulation of internal representations by an artificial real-time perceptual stream, here configured as a kinematical determined acoustical stream.

\section{Action determined perceptual streams}

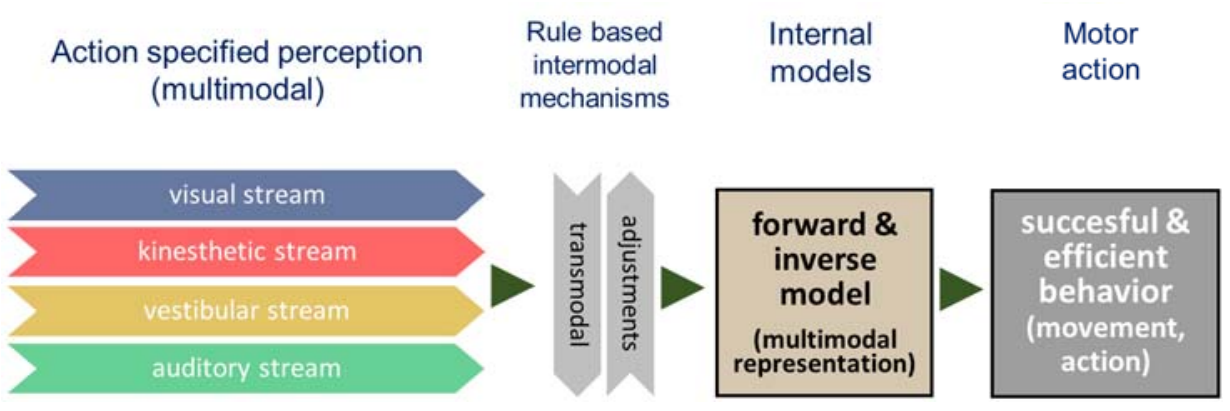

Fig. 1a. Action-specified perceptual streams of different modalities are aligned and integrated in the perceptual system (multisensory areas) configuring internal models 


\section{Action determined perceptual streams}

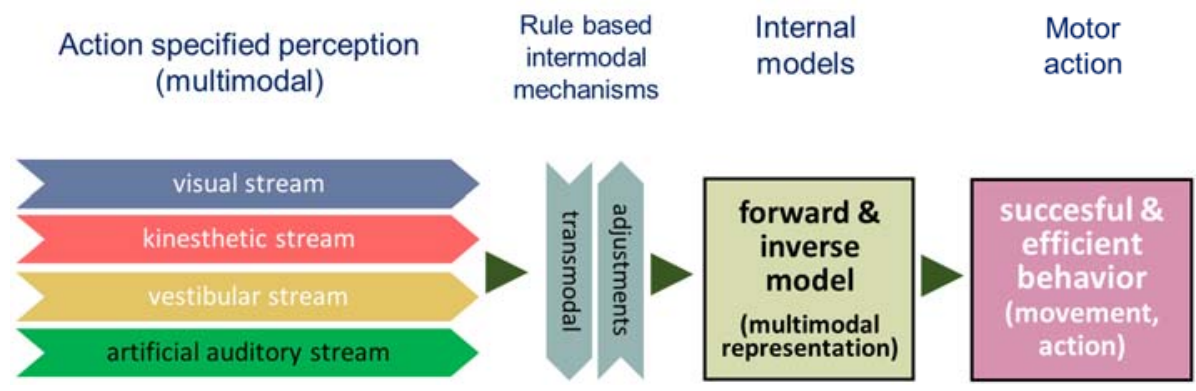

Fig. 1b. Changing one perceptual streams will change transmodal adjustments and multisensory integrations and thereon modify emergent internal models - enabling the modification of model-based movement patterns. Change of colors indicates a change within the referenced process (auditory stream, intermodal mechanisms, internal model, motor action)

With figures $1 \mathrm{a} \& 1 \mathrm{~b}$ the emergence of internal models based on the combined perception of different modalities is illustrated. A specifically shaped internal model can only emerge based on specifically shaped perceptual streams, which is given by the action just executed: Perceptual streams are modulated by the specific movements of the action, as described for the specification of the optical flow from the owns movement by Gibson $(1966,1976)$ within the visual domain. Based on the idea of direct perception and direct perception-action coupling of Gibson, Stoffregen and Bardy (2001) developed the idea of a seamless unity of the different perceptual modalities into a gobal array postulating intensitive intermodal processing of information and multimodal integration. Amodal or supramodal representations are resulting from intermodal/multisensory processing and integration. This construct is based on the idea of a unified perceptual system consisting of different channels with their sensors directed to the distribution of ambient energy (light, sound, gravity etc.). The current constellation of the subject and the environment is specified by the distribution of energy in the different modalities, which addresses the adequate receptors in a specific manner. Whilst this specification is not unambiguous within a single perceptual modality, unambiguity is reached on the level of complete integration (global array).

Concerning the development of more efficient methods for the support and enhancement of interactive multi-agent scenarios (joint action, social interaction, interpersonal coordination) it is essential to know about these interrelations, because the establishment of interpersonal coordination is depending on common knowledge of the action goal (Vesper, Butterfill, Knoblich and Sebanz 2010, Keller, Novembre and Hove 2014, Miyata, Varlet, Miura, Kudo and Keller 2017). Besides the structural interrelations which determine also in parts how information is interactively processed it is important to know what kind of information is being processed. After many decades of modality 
specific research within the last three decades the relevance of multisensory and intermodal mechanisms concerning the regulation of behavior has been taken into account more and more by neuroscientists (Stein and Meredith 1993, Calvert, Spence and Stein 2004, Spence and Driver 2004, Baumann and Greenlee 2006, Shams and Seitz 2008, Spence 2015). Multisensory integration and intermodal processing of sensory information are key mechanisms for an efficient use of additional auditory information which should make it possible in the future to shape internal representations and processes of motor control and motor perception directly. Some basic findings from multisensory integration research should be recalled here. To support the understanding of the observed and assumed efficiency of an anticipative auditory kinematics it is important to know that

o anticipated and perceived sensory/perceptual effects are available in different perceptual modalities during the planning and execution of action (Zmigrod \& Hommel 2013),

o different perceptual streams are continuously integrated seamlessness down to the level of a single neuron (Stein \& Meredith 1993, Stein \& Stanford 2008), and

o that perceptuomotor internal models are consisting partially of multisensory/intermodal representations (Berthoz \& Viaud-Delmon 1999, Stoffregen \& Bardy 2001, Lacquaniti, Bosco, Gravano, Indovina, La Scaleia, Maffei et al. 2014).

Based on this knowledge the configuration of an additional anticipative auditory kinematics e.g. for enhancing the perception and anticipation of movement kinematics in single as well as dual-agent scenarios is an interesting issue. Multisensory information is more effective on motor perception, motor control and motor learning (Seitz, Kim \& Shams 2006, Shams \& Seitz 2008) as well as relearning in motor rehabilitation by (Johansson 2012, Schaefer 2014). Also from our own research group there is some evidence that individual motor behavior can be supported effectively by additional kinematic real-time sonification (motor learning: Effenberg, Fehse, Schmitz, Krueger \& Mechling 2016, motor rehabilitation: Schmitz, Kroeger \& Effenberg 2014, character acquisition: Effenberg, Schmitz, Baumann, Rosenhahn \& Kroeger 2015). Furthermore, the identification of one's own movements against others can be supported by kinematic sonification (Schmitz \& Effenberg 2016) and interpersonal coordination can be enhanced (Schmitz \& Effenberg 2012).

Besides, Schmitz, Mohammadi, Hammer, Heldmann, Samii et al. (2013) found evidence that the action observation system of humans can be addressed by additional kinematic sonification: This findings can serve as a plausible explanation for the related bahvioral effects, but moreover beyond that: In future kinematic sonification if generated structurally equivalent to the visual kinematics, even the perception and interaction with artificial systems like humanoid robots might get supported. Audiovisually integrated percepts could make robots behavior easier understandable and more reliable predictable by human co-actors - even without the need of conscious cognition. Perceiving the kinematics of the co-actor audiovisually might enhance the smoothness of 
human-robot cooperation by supporting internal simulations as a key element of fluent interpersonal coordination (Khoramshahi, Shukla, Raffard, Bardy \& Billard 2016). This idea is supported by former work of (Lahav, Saltzman \& Schlaug 2007, Kohler, Keysers, Umilta, Fogassi, Gallese \& Rizzolati 2002, and Keysers, Kohler, Umilta, Nanetti, Fogassi \& Gallese 2003), emphasizing the relevance of multimodal integration of the perceptual streams also via audiovisual mirror neurons as well as of intermodal binding mechanisms (Calvert, Spence \& Stein 2004).

Turning the focus explicitly to interactive dual-agent scenarios, additional auditory kinematics applied to both agents can enable common audiovisual perception of agents movements and thereby a more precise interpersonal temporal and spatial coordination can be expected. It is to assume that audiovisual kinematic movement information can be used to provoke an enhanced interactive temporal coupling as described by (Oullier, De Guzman, Jantzen, Lagarde \& Kelso 2008) for visual settings: The authors reported a relative phase and frequency overlap between movements of individuals when acting together. As a consequence, interpersonal coordination should get smoother and more reliable when interaction/coordination is based on multisensory audiovisual information. Redirecting the gaze to the field of music as an interesting field of interpersonal sensorimotor coordination, timing, synchronization and aesthetics within an orchestra are referring to the causal relationship of the movement kinematics of violinists and conductor(s), as shown by (D'Ausilio, Badino, Li, Tokay, Craighero, Canto, Aloimonos \& Fadiga 2012). Keller, Knoblich \& Repp (2007) demonstrated that sensorimotor synchronization as well as the recognition of self-played piano sequences is based on internal action simulation. Sensorimotor synchronization is a determinant of the interaction between humans as well as in human-robot interaction, as pointed out by (D'Ausilio, November, Fadiga \& Keller 2015). Taken together results indicate that internal simulation of others actions is an important mechanism for understanding of - and the smooth participation in - cooperative actions (Bishop and Goebl 2018). Obviously internal simulation of coordinated action is not dependent on visual information and the visual mirror neuron system: Internal simulation can be also initiated by auditory information addressing auditory/audiovisual mirror neurons, if acoustics is directly linked to the related movements. This is given when playing music instruments and it is equally given for appropriate kinds of kinematic movement sonifications.

Besides interpersonal coordination in music there is comprehensive evidence on several determinants of joint action like joint attention, action observation, task sharing, action coordination, and agency by Sebanz, Bekkering and Knoblich (2006), and with reference to rocking chair movements by Goodman, Isenhower, Marsh, Schmidt \& Richardson (2005). In this field of research authors emphasize the importance of the ability to predict the actions of others (the what, when, and where of others' actions) for a smooth and successful interpersonal coordination on joint actions (Sebanz and Knoblich 2009). Referring to the concept of planned and emergent coordination from Knoblich, Butterfill and Sebanz (2011) auditory information is especially suitable for the support of emergent coordination due to its fast and unconscious perceptuomotor processability. Thereby emergent auditory/intersensory coordination should support 
fast and precise interpersonal perception-action couplings. Demos, Chaffin, Begosh, Daniels \& Marsh (2012) already showed that perceiving another participant moving in a rocking chair subconsciously influences one's own rocking frequency, even if both participants are instructed to ignore each other. Both modes - seeing and hearing the other person rock - elicited spontaneous coordination, but it is remarkable, that hearing amplified the visual effects. Obviously, participants unintentionally integrate the perceived movement frequency into their own movement production - a phenomenon supporting the concept of inter-corporeality. Music, in an additional condition, serves as social glue: participants, who synchronized more with music felt more connected (Demos et al. 2012).

Summing up: From a traditional point of view, perception, prediction and action referred to different phenomena guided by own rules each and based on separated functions. More current theories now state that one aspect cannot be considered without the others. For example, a key hypothesis in embodiment and joint action research is that action observation triggers an internal modelling of movements which actively involves the motor system. Therefore, during interpersonal interactions, the motor system seems to fulfill two distinct functions: one related to observing, simulating and anticipating other persons' actions and another related to planning and controlling one's own motor behavior. Some evidence was presented that auditory movement information - as on music making or movement sonification - is usable as an additional common reference framework for both interaction partners, enhancing temporal and spatial determinants of cooperative actions. Inter-corporeality, interpersonal coordination and even interkinesthesia in sports as well as in everyday scenarios are based on a close connection of perception and anticipation of action as well as on common motor actions of all agents being involved in interpersonal coordination such as in team-rowing. It should have become clear, that the anticipation of co-actors/co-agents actions and intentions is an essential element of smooth and successful interpersonal/inter-agent coordination.

Whilst the first section deals with the emergence of motor behavior with a focus on auditory information on a low-granular level, the 2 . Section is dedicated to a finer granular level: Here adaptation modules and the references to different perceptual modalities are taken into consideration mainly. Section 3 focusses on rhythmic auditory cueing and its influence on neurophysiological activations. Furthermore, we discuss how these sensory stimulations might shape the perceptuomotor representations during rehabilitation of movement disorders. Based on the first three sections the 4. Section is dedicated to the technological side of kinematic real-time sonification as a powerful instrument to support perceptual-motor processes and modulate emergent perceptuomotor behavior specifically.

\section{Sensorimotor Adaptation and Learning Aspects}

Although sport scientific research predominantly focusses on the analyses of realworld like tasks, important insights into mechanisms of motor learning can be gained 
by fundamental research in well controllable, experimental settings with relatively simple movements. According to Haar, Donchin and Dinstein (2015), paradigms on sensorimotor adaptation belong amongst the most fundamental approaches for understanding motor control. The following section will describe adaptation mechanisms and how internal models seem to be linked to sensory modalities and effectors.

\section{Approaches and Mechanisms}

The majority of adaptation studies focusses on hand, arm or eye movements. Eye movements represent an attractive object of research, because they have only few degrees of freedom and are well describable. They are predominantly linked to visual perception, but they also exert influence on other perceptual modalities as well as movement effectors. For example, is has been shown that eye positions or eye movements affect spatial representations of sounds (Lewald \& Getzmann, 2006) and internal models for hand and arm movement control (Dijkerman, McIntosh, Anema, de Haan, Kappelle \& Milner, 2006; Schmitz \& Grigorova, 2017). Thus, they have to be considered as important for perceptions and nearly all types of movements. Accordingly, fundamental contributions to the understanding of motor learning have been achieved by research on blink conditioning and saccadic adaptation to target displacements (doublesteps).

Smith, Ghazizadeh and Shadmehr (2006) observed that already metrics of simple eye movements are not altered by a single adaptation mechanism, but by several mechanisms acting concurrently. A few years later the same principle was shown for hand movements (Lee \& Schweighofer, 2009), which helped to refine the understanding of internal models and their multiple subfunctions. An important insight was that internal models seem to be composed of further sub-units termed 'modules'. Modules specify spatial and force-related parameters of movements and can be more or less flexibly arranged during the generation of motor commands (Bock, 2013; Wolpert, Diedrichsen \& Flanagan, 2011). By observing that adaptation transfers between hands (Sainburg \& Wang, 2002), between hand pointing and walking movements (Morton \& Bastian, 2004) or between eye movements and hand movements (Bock, Schmitz \& Grigorova, 2008), it was shown that internal models are rather effector-independent than effectorspecific, i.e. the same internal model can by linked to different movement effectors. By showing that transfer between effectors is not obligatory but fragile as it happens in some but not other conditions, it was concluded that internal models are flexibly linked to effectors by a weighting or a switching function (Bock, 2013).

\section{Generalization Between Visuomotor and Audiomotor Adaptation}

Whereas effector-specificity of internal models has been investigated in depth, less work has been performed with respect to the role of sensory modalities. One of the first researches who investigated whether adaptation in one modality transfers to another modality was Samuel Harris (Harris, 1963, 1965). He reported that adaptation of arm movements achieved with prism glasses transfers fully to pointing movements performed with closed eyes to sound sources. The finding of fundamental transfer from the visual to the auditory modality has been confirmed several times up to now (Cohen, 
1974; Kagerer \& Contreras-Vidal, 2009; Mikealian, 1974; Michel, Pisella, Prablanc, Rode, \& Rossetti, 2007) and can be regarded as validated scientifically. As Harris further found an altered position sense of the trained but not the untrained arm in the same experiments, he concluded that the position sense of the trained arm is the locus of adaptive changes. These changes would affect every arm action, independently from whether the action is performed in a visual or an auditory condition or with eyes closed.

Craske (1966) developed a simple acoustic adaptation paradigm, in which participants pressed a button below sound sources which triggered another sound from a loudspeaker 12 deg away. Repeated practice induced pre-post changes in an auditory and in a visual localization task performed with the trained arm. According to Harris (1965), the author concluded that the position sense of the trained arm had adapted. However, other results do not comply with this conclusion: Mikaelian (1972) adapted arm movements while participants continuously moved a sound source in front of the body from side to side while wearing pseudophones. These are headphones connected to rotatable microphones above the head. A rotation of the microphone axis should mimic interaural time differences with respect to the sound source in the hand. This author found audiomotor aftereffects during movements with the trained, but also with the untrained arm. Most importantly, he did not find visuomotor aftereffects, why he concluded that not the position sense but auditory perception had adapted.

Nevertheless, since then changes induced by sensorimotor adaptation have been regarded as sensory changes, and sensorimotor adaptation has been discussed in line with models on multisensory fusion and intersensory binding, accordingly (Chen \& Vroomen, 2013; Welch, 1978). After the pioneer studies in the 60s and 70s, audiomotor paradigms had been abandoned from research on hand and arm movement adaptation for a long time or not been published, presumably until the work from Oscari, Secoli, Avanzini, Rosati and Reinkensmeyer (2012). However, researchers broadened the overall picture by analyzing proprioceptive effects of prism adaptation. Bernier, Gauthier and Blouin, (2007) let participants point to their unseen index finger of the untrained hand before and after prism adaptation. No pre-post changes became evident and these as well the authors who cited this research concluded that proprioception had not been altered by visuomotor adaptation. However, very recently another study was published, which reported significant changes of the proprioceptive position sense after adaptation to a visuomotor rotation (Flannigan, Posthuma, Lombardo, Murray \& Cressmann, 2018). This suggests that proprioceptive adaptation might depend on the applied adaptation paradigm. Another possible explanation is that Bernier et al. (2007) did not test for changes in the position sense of the un-trained arm - this would have been necessary for the validity of their conclusion, because some studies indeed report this effect.

\section{Perceptual, Motor or Sensorimotor Recalibration?}

Although it seems to be plausible, several studies give rise to doubts on the hypothesis of perceptual adaptation. First of all, it seems possible to induce purely motor aftereffects (Magescas \& Prablanc, 2006). Second, Hatada, Miall and Rossetti (2006) 
reported that visual and proprioceptive aftereffects of a single adaptation session last for several days, which is quite astonishing when participants continue their regular daily activities in the days after the experiment which should induce de-adaptation or recalibration of the perceptual changes back to normal. Furthermore, the authors observed that proprioceptive aftereffects measured during passively performed movements decayed after two days, whereas proprioceptive aftereffects persisted for seven days when movements were performed actively. Therefore, an alternative to the hypothesis of perceptual changes has to be derived: One explanation might be the adaptation of internal models that are flexibly linked to perceptual and motor processing. In the following, we describe a framework that accounts for this hypothesis.

\section{Connectivity of internal models to sensory modalities}

According to the MOSAIC model for sensorimotor control from Wolpert and Kawato (1998), the sensorimotor system contains of multiple parallel internal models. However, concurrent adaptations to only two sensorimotor discordances (dual adaptation) have been shown to interfere with each other, suggesting a bottle neck in the sensorimotor system during adaptation. Interference of two adaptive processes complies with the hypothesis on perceptual recalibration, because it is plausible that one sensory modality cannot have two incompatible states at the same time. Nevertheless, under some conditions, dual adaptation seems to be possible without costs. One condition is the adaptation of two arms. First Prablanc, Tzavaras and Jeannerod (1975) and later other authors (e.g. Galea \& Miall, 2006; Martin \& Newman, 1980) showed that the left arm can adapt to one sensorimotor discordance while the right arm adapts concurrently to an opposite directed sensorimotor discordance. In such a case, it is interesting to analyze what changes occur in which sensory modality. Given the assumption that one sensory modality cannot adapt at the same time to opposite directed distortions of space, dual arm adaptation would be compatible with adaptation of the arm specific position senses, only.

Therefore, Bock and Schmitz (2013) tested, whether movements performed to visual targets with clockwise rotated visual feedback for the left arm and counterclockwise rotated feedback for the right arm transfers to movements performed to auditory targets without feedback. As shown before by other studies, both arms adapted successfully and revealed significant pre-post changes of the trajectories to visual targets. Most interestingly, adaptation transferred by $66 \%$ to the auditory modality, which is substantial but incomplete. This effect occurred in each arm. Therefore, it is not compatible with adaptation in the visual modality, but with a higher weighting of visual compared to auditory information after dual visual adaptation. Further work from the same authors (Schmitz \& Bock, 2014, 2017) showed that the visual modality is not per se rated higher than the auditory modality. When arm movements adapt in an audiomotor paradigm, i.e., participants perform arm movements to auditory targets with auditory real-time feedback about movement trajectories, adaptation (after-)effects are larger in the auditory compared to the visual modality. Thus, it is always the trained modality that gets larger (or similar) input weights than the untrained modality, irrespective of whether it is the visual or the auditory modality. This is illustrated by the schematic model in 
Figure 1, whose basic features had been developed by Bock (2013) and specified in further work together with Schmitz (e.g. Schmitz \& Bock, 2014). Adaptation of the right arm to an audiomotor discordance recalibrates one internal model (IM 2), and specifies the weighting function W4 which gets larger than W3. At the same time, the right arm can adapt to an audiomotor discordance which recalibrates another internal model (IM 1) and enlarge W2 compared to W1 or to a visuomotor discordance which trains IM 1 with similar efficiency but enlarges W1 compared to W2 (Schmitz \& Bock, 2017).

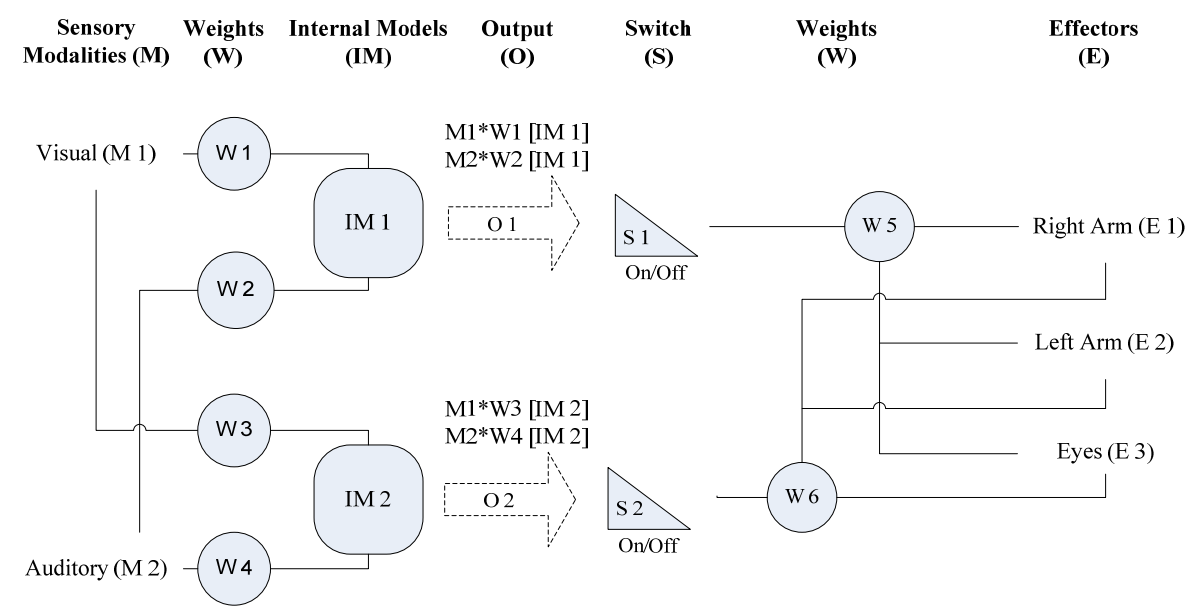

Fig. 2. Essence of the studies from Bock \& Schmitz (2013), Grigorova and Schmitz (2017) and Schmitz and Bock $(2014,2017)$ as amendment of the model proposed by Bock (2013). Internal models are considered to be amodal and connected by weighting functions (W1-W4) to sensory modalities (M1, M2). Outputs (O1, O2) from internal models (IM1, IM2) pass two-state switches (S1, S2). If both switches are 'on', the outputs from IM1 and IM2 are added (e.g. M2*W2 [IM 1] plus M2*W4 [IM 2]). These outputs are weighted (W5, W6) before sent to different effectors. All colored components are specified during sensorimotor adaptation and learning.

\section{Connectivity of internal models to different effectors}

The schematic model allows to explain further phenomena related to sensorimotor adaptation and learning. Both internal models are connected to several effectors. Their outputs are weighted again (W5 and W6), which results in full or partial intermanual transfer after adaptation (Imamizu \& Shimojo, 1995; Michel et al., 2007). Furthermore, it accounts for transfer of adaptation from hand movements to eye movements and vice versa (Bock et al., 2008). The connection of IM 1 and IM 2 to several effectors also explains interference within hand or arm motor systems (Wigmore, Tong \& Flanagan, 2002) or between hand and eye movement adaptation, when adapting to opposite directed spatial discordances (Schmitz \& Grigorova, 2017). 
Interference between two conflicting internal models can be inhibited. Partial inhibition seems to be possible on the basis of cognitive executive inhibition. This is indicated by reports on facilitation of adaptation to two opposite directed sensorimotor discordances through explicit knowledge about these discordances (Imamizu, Sugimoto, Osu, Tsutsui, Sugiyama, Wada, et al., 2000) as well as by significant correlations between the amount of interference and participants' mental flexibility and cognitive inhibition abilities (Schmitz, 2016). Whereas these effects occur fast and are short-term, complete inhibition seldom occurs spontaneously. Instead, it seems to be achieved through training of slow-acting mechanisms in several hundreds to thousand trials (Schmitz \& Grigorova, 2017). Wolpert et al. (2011) describe it as the problem to assign effectors and internal models. Bastian (2008) regards it as sensorimotor learning. In the model presented in Figure 1, it is a decoupling of internal model and effectors through training of the context dependent on/off-switch functions S1 and S2. As long as the switch functions have not been established, yet, the output of the two internal models is fused nearly linearly. Most interestingly, the weighting of modalities (W1-W4 in Figure 1) is still relevant so that adaptation transfers with these modality-specific weights between effectors (Schmitz \& Bock, 2017).

\section{Conclusion}

Taken together, auditory feedback of arm movements seems to address the same mechanisms for sensorimotor adaptation as visual feedback. Therefore, spatial auditory and visual feedback can substitute each other. Further data suggest that the best effect is achieved when the feedback modality corresponds to the target modality (Schmitz, 2014), probably because trajectories are first specified within a modality before the trajectory specifications of different modalities are fused to achieve an optimal outcome (Oostwoud, Wijdenes \& Medendorp 2017; Tagliabue \& McIntyre, 2014).

Internal models seem to be neutral with respect to sensory modalities (amodal) and to be coupled by default with sensory and motor instances of the sensorimotor system. This becomes evident from multiple findings on intermodal as well as interlimb transfer. During sensorimotor training, the sensorimotor systems alters linkages between sensory modalities and internal models as well as between internal models and effectors (semi-)permanently by specifying weighting functions. Furthermore, extensive training is required to learn the de-coupling of an internal model and an effector, which allows efficient switching between different adaptive states subsequently. This might rather be termed sensorimotor learning than adaptation.

Adaptive changes might be less based on recalibration of sensory percepts than on recalibration of internal models. Theory states that the output from internal models is used to generate feedforward expectations about the sensory consequences of the intended actions, which are compared with the actions' actual outcome in a next step. Shadmehr, Smith and Krakauer (2010) state that feedforward expectations are fused with sensory percepts and thereby affect the overall percept. This might explain how adaptation alters spatial perception without recalibrating sensory modalities. 


\section{Effects of Auditory Information on Cyclic Movements}

Pre-determined auditory stimuli guiding cyclic movements have been well documented in published literature. This type of stimulation intuitively interconnects movement with rhythmic stimulations due to widespread inclination towards music. This results in influencing and coordinating movement patterns while timing the specific mechanisms and therefore initiating a common neural network. Such auditory rhythmic patterns can impart great neurophysiological and behavioral changes during motor performance. Rhythmic auditory stimulations have been reported in the published literature to allow benefits in motor execution (Ghai, Ghai \& Effenberg, 2017; Ghai, Ghai, Schmitz \& Effenberg, 2018), motor control (Thaut, Schleiffers \& Davis, 1991), interpersonal coordination (Keller, Novembre \& Hove, 2014), and breathing (Murgia et al., 2016).

Rhythmic auditory cueing exploits the intricate neuroanatomical relationship in between the auditory and motor centers especially extending towards cortical, subcortical structures, brainstem and alpha motor neurons of spinal cord (Felix, Fridberger, Leijon, Berrebi \& Magnusson, 2011; Thaut, 2005). The auditory system is quite fast, precise and efficient in detecting anomalies in auditory stimuli, and developing stable temporal templates (Thaut, 2003). Likewise, auditory cortex has been reported to perceive stimuli with shorter reaction times $(20-50 \mathrm{~ms})$ as compared to its visual or tactile counterparts (Nombela, Hughes, Owen \& Grahn, 2013). Moreover, Fujioka, Trainor, Large \& Ross (2012) reported modulation of neuromagnetic $\beta$ oscillations with rhythmic auditory stimuli in auditory cortex, cerebellum, inferior frontal gyrus, somatosensory area and sensorimotor cortex. The stimuli have been suggested to activate inferior colliculi (Tierney \& Kraus, 2013), cerebellum, brainstem (Debaere, Wenderoth, Sunaert, Van Hecke \& Swinnen, 2003; Hausdorff et al., 2007), sensorimotor cortex (Asanuma \& Keller, 1991; Suh et al., 2014), further instigating reorganization in cortico-cerebellar circuits (Luft et al., 2004). Rhythmic auditory stimuli have also been suggested to reap the benefits of the preserved neural centers during rehabilitation and even learning (Torres, Heilman \& Poizner, 2011) (see also "kinesia paradoxica" (Rinehart et al., 2006)). Studies report that motor activities directed by external sensory cueing evoke pathways via cortical, premotor areas (Ghai et al., 2018), effectively bypassing the affected basal ganglia region (Young, Rodger \& Craig, 2014), which usually is affected in motor dysfunctions. Studies have suggested that rhythmic sensory cues can also replace deficient pallidal-cortical projections and serve a signal to supplementary motor area, like a feed-forward input for aiding motor task, thereby reducing bradykinesia, and associated motor deficits (Cunnington, Iansek, Bradshaw \& Phillips, 1995). Similarly, the external cueing can supplement critical spatio-temporal information which is necessary for initiation or facilitating a motor activity (Nieuwboer et al., 2009; Nieuwboer et al., 2007), such as during gait or arm movements (Whitall et al., 2011). Likewise, recent research suggests that rhythmic cueing might also assist in facilitating frontal-lobe cognitive strategies (working memory) by externally cueing the motor execution (El-Tamawy, Darwish \& Khallaf, 2012). According to Campos-Sousa, Campos-Sousa, Ataide, Soares \& Almeida (2010) frontal lobe areas can select a motor program in response to an external stimulus, and send it to the primary motor cortex, which are mainly guiding such movement patterns. External cues also contribute to 
sensorimotor integration that requires organizing and processing of proprioceptive inputs in working memory (Ghai, Schmitz, Hwang \& Effenberg, 2018). In context of gait execution, the external rhythm can guide the patients to synchronize their ground contact and lift-off times (Ford, Malone, Nyikos, Yelisetty \& Bickel, 2010). The patterns might also serve as a medium for assisting in planning before executing a movement (Thaut et al., 2007). Moreover, the periodicity in rhythmic auditory feedback has also demonstrated to effectively reduce variability in musculoskeletal activation patterns, thereby allowing more economical and consistent motor unit recruitment (Thaut, 2005), further smoothing the velocity and acceleration profiles of joint motions by scaling movement time (Thaut, 2005).

\subsection{Rehabilitation Applications}

Recently conducted systematic reviews and meta-analyses by our department to analyze the effects of rhythmic auditory cueing on gait performance (a cyclic task) among fall prone population groups revealed interesting findings. First and foremost, beneficial effects were reported for rhythmic auditory cueing for enhancing gait stability and performance in patients affected from parkinsonism (Ghai et al., 2018), cerebral palsy (Ghai et al., 2018), and in aged population groups (Ghai et al., 2017). Considerable enhancements in terms of small-to-large effect sizes in spatiotemporal gait parameters were reported for all population groups. However, cadence amongst the parkinsonian patients was the only spatiotemporal parameter that saw a negligible difference. Typically, patients with parkinsonism exhibit a shuffling gait, where cadence is usually high and step length is small. Here, a reduction in cadence with the delivery of rhythmic auditory cueing suggests that the stimuli instead of increasing the gait execution, promoted more automaticity, and hence stability during its execution.

Furthermore, modulation of differential factor in rhythmic auditory stimulation have been suggested to impact different aspects of motor execution. For instance, change in tempo has been associated with various neurophysiological changes such as, increased neuronal activation in fronto-occipital networks (Thaut et al., 2009), excitability of the spinal motor neurons by reticulospinal pathways, which might possibly reduce the response time for a motor task. Likewise, variation in tempo during training is suggested to be beneficial for maintaining a healthy gait pattern, as constant rhythmic pattern for longer durations have shown to decrease fractal scaling of stride times from healthy $1 / \mathrm{f}$ structure, possibly because of organization of stride time variability around a single frequency (Delignières \& Torre 2009; Hausdorff et al., 1996; Hove, Suzuki, Uchitomi, Orimo \& Miyake, 2012). Additionally, Buchecker, Wegenkittl, Stöggl, and Müller (2017) demonstrated beneficial effects of enhanced variability within training on posture and electromyographic activity. This might serve to be beneficial for parkinsonian patients to learn how to regulate gait, when passing through fall-prone environments. Moreover, the induction of variability can also be subjected subliminally (for instance changes in tempo, interstimuli interval, see also (Tecchio, Salustri, Thaut, Pasqualetti \& Rossini, 2000)). This might maintain variability in the rehabilitation protocol and simultaneously prevent any conscious stress to excessively speed up or slow down the gait. However, Hopkins, Kass, Blalock and Brill (2017) reported that cross modal cueing can avoid information overload in the native sensory modality by directing taskirrelevant information towards the underused sensory modality (Hameed, Ferris, 
Jayaraman, \& Sarter, 2009). Here as well, the introduction of auditory feedback could have possibly allowed enhancements in repositioning accuracy by information in the sister domain (Ghai et al., 2017; Lohnes \& Earhart, 2011).

Finally, recent research also points out the beneficial effects of engaging biologically variable rhythmic auditory cueing as compared to isosynchronous cueing. Since, patients with parkinson's, stroke, aged population groups are characterized by a higher threshold for action relevant acoustic input, therefore using ecologically valid action related sounds convening spatio-temporal information can possibly enhance saliency of sensory information, transferring spatio-temporal information effectively and therefore providing more benefits (for a detailed review see (Ghai et al., 2018)). For instance, here timbre of an auditory input at a higher intensity merged in a broad ascending melody and a rich harmony can possibly motivate a patient to exert more power.

\subsection{Referencing on the Internal model theory}

Auditory cueing is meant to enhance and shape multisensory representations and address components of auditory motor processing. As mentioned before, this type of acoustic feedback works independently of conscious cognitive processing. This might allow an enhanced spectrum of movement information based on instructional information. Thereby, supporting the development of sensorimotor or perceptuomotor representations. According to the internal model theory of Wolpert, Ghahramani \& Jordan (1995) this entrainment effects might help in reducing sensory error estimation during the motor planning phase, further resulting in an efficient movement execution (Wolpert, Diedrichsen \& Flanagan, 2011). Here the instructive information can be possibly used to support the development of forward models.

Rehabilitation intervention with movement sonification is still a rather unexplored territory. Concerning stroke rehabilitation there is first evidence that on hemiparesis of the upper limbs real-time kinematic movement sonification can enhance the effectiveness of a traditional method (Schmitz et al., 2014). Moreover, recent research by Effenberg et al., (2016) has demonstrated that the effects of movement sonification extend the benefits offered by rhythmic auditory stimulations. Therefore, the current section lays possible interpretations for future studies as to how movement sonification might influence behavior and motor performance in movement disorders. The following sections explain the theoretical, experimental and technical aspects of how such online auditory feedback might shape these movement representations in real life settings.

\section{Generation of Artificial Auditory Information}

\subsection{Digital Sound Generation}

This subsection is to explain the process of generation of digital sound and its considerations. Auditory information can be generated by a speaker system connected to digital devices (e.g. computer, smartphone). A host system transmits control signals to a speaker. Referring to the control signal, an amplifier drives the mechanical vibration of the magnet and the voice coil in the speaker. The vibration is transmitted to the environment of the speaker through a speaker cone which is set into vibration, changing 
the pressure of the ambient air. Pressure changes between frequencies of about $20 \mathrm{~Hz}$ to $20 \mathrm{kHz}$ are hearable for the human ear as sound. Sound can also be transmitted as waves in certain materials (e.g. air, water, metal, wood). When sound reached the human ears, vibrations are transmitted to the eardrum: Acoustic pressure changes are transformed into electrical nerve impulses and transmitted to auditory and multisensory areas in the brain. When auditory information is regarded as a perception of small movements on the eardrum, it can be compared to a perception of body movement in terms of energy transmission.

\subsection{Relationship of Auditory and Movement Parameters}

This subsection discusses the selection of suitable sound parameters to generate effective auditory movement information. Electronic sound synthesis offers a huge number of sound parameters and sound effects (e.g. pitch, loudness, timbre, panning effect), which can be combined to movement parameters in a certain manner. This allows a vast number of mappings between sound and movement. If the auditory information should be processed immediately, the sound should be perceivable intuitively. Natural kinetic-acoustic relationships have been taken as a base for intuitive perception (Effenberg, 2005; Dubus, Bresin, 2013). The pitch has been used as the most used auditory parameter in mappings such as 'kinetic energy to pitch' and 'location to pitch' (Dubus, Bresin, 2013). Depending on biological and psychological factors, effects of the auditory feedback alter in different situations. For example, hearing ability, movement ability, fatigues, and mood can influence of the connection between sound and movement. Although there are a large number of possible mappings, our approach is mapping potential and kinetic energy to sound energy, which can guide at least direction and the amount of alterations in mapping. Considering the fact that human movements consist of rotational movement, the energy equation can be expressed with potential energy $E_{p}$ and rotational kinetic energy $E_{k}$. The equations of total energy $E_{P . t o t a l}$ is as below:

$$
E_{P . t o t a l}=E_{p}+E_{k}=m g h+1 / 2 I \omega_{m}^{2}
$$

where $m$ is mass, $g$ is gravity, $h$ is the height, $I$ is rotations inertia (comparable to mass in linear kinetic energy equation) and $\omega_{m}$ is the angular velocity. In the last equation, the first term is potential energy and the second term is the kinematic energy. When potential energy is constant, the total energy is proportional to $\omega_{m}{ }^{2}$. In terms of the sound energy $E_{A}$, the equation is the integration of sound energy density over the volume as below:

$$
E_{\text {A.total }}=E_{A . p}+E_{A . k}=\int_{V}\left(p^{2} / 2 \rho_{0} c^{2}\right) d V+\int_{V}\left(\rho v_{p}^{2} / 2\right) d V .
$$

As shown in Eq. (2), sound energy consists of potential energy $E_{A . p}$ and kinetic energy $E_{A . k}$. The equation is expressed with given parameters ( $V$ : the volume of interest; $p$ : sound pressure; $v_{p}$ : particle velocity; $\rho_{0}$ : the intrinsic density of the medium; $\rho$ : the local density of the medium; $c$ : the speed of sound) (Müller \& Moeser, 2012). When a tiny local volume is considered because of the size of human ear, $E_{\text {A.local }}$ can be obtained from the differential of Eq. (2) about the volume as below: 


$$
E_{\text {A.local }}=p^{2} / 2 \rho_{0} c^{2}+\rho v_{p}^{2} / 2 \text {. }
$$

As other parameters $\left(\rho_{0}, \rho, c\right)$ are given from the environment, the parameters can be constant. Finally, the equation is become as below:

$$
E_{P}=A h+B \omega_{m}{ }^{2}=C p^{2}+D v_{p}{ }^{2}=k E_{A . s p o t}
$$

where, $A, B, C, D$, and $k$ are coefficients. According to Eq (4), an audio system can change the amplitude (function of $p$ ) and frequency (function of $v_{p}$ ) in order to control sound energy. Audio amplitude or frequency can express the movement energy change caused by the angular velocity or the height. Although personal use of movement energy determined by various factors, based on this simple equation, it is helpful to design a number of possible mapping between two auditory and two kinematic parameters. With the base design, fine tuning is possible as well as exaggerated and reduced auditory effects are also available.

Auditory Perception of Loudness As another consideration in designing sound, this subsection deals with logarithmic human auditory perception about amplitude. In the waveform of a signal, the amplitude is defined on as the peak value in a period. Audio amplitude is regarded as loudness of sound, which is related to sound pressure. Sound pressure is defined in the international system of unit (SI unit) in Pascal (Pa), and it is the deviation between local sound pressure and atmospheric pressure without sound (equilibrium state). For express loudness, decibel (dB) is defined, which is logarithmic function of a ratio of two factors: The human ear feels loudness relatively to the equilibrium state, and human auditory perception is logarithmic. The following equation shows the relationship between decibel and the ratio of the measured power $(P)$ and the reference power $\left(P_{0}\right)$ :

$$
L_{p}=10 \log _{10}\left(P / P_{0}\right) d B
$$

where $L_{p}$ is the ratio expressed in decibel. From Eq. (5), when $P$ becomes ten times of $P_{0}, L_{p}$ is $10 \mathrm{~dB}$ higher. Whenever loudness is $10 \mathrm{~dB}$ higher, the human ear perceives the same loudness difference. From Eq. (4), the sound power (derivative of energy with respect to time) is proportional to the square of sound pressure. The equation of $L_{p}$, sound pressure $\left(p_{A}\right)$, and reference sound pressure $\left(p_{A .0}\right)$ is as below:

$$
L_{p}=20 \log _{10}\left(p_{A} / p_{A .0}\right) d B
$$

As shown Eq. (6), humans can perceive the same difference when $p_{A}$ is $\sqrt{10}(=3.162)$ times more than $p_{A .0}$. For appropriate auditory feedback, loudness should be above the hearing threshold $(0 \mathrm{~dB})$ and clearly below the pain threshold $(120 \mathrm{~dB})$. An effective auditory feedback should be displayed between about $30 \mathrm{~dB}$ (calm room) and $80 \mathrm{~dB}$ (limit to avoid hearing damages under long time exposure). Though the level of decibel is affected by the basic level of amplitude, the distance from the sound source and the displayed frequencies, an effective and pleasant movement sonification should be configured carefully and the loudness should be measured just near to the ears. 


\subsection{Auditory Perception of Pitch}

This subsection is related to the nonlinear characteristics of auditory perception concerning audio frequency and musical harmony. When mapping pitch to kinematic parameters, especially when two or more sounds are displayed simultaneously. This provides listeners with the same effect in different situations and lead them to feel pleased with auditory feedback. Human auditory perception of audio frequency is relative to a logarithmic function. An octave is defined as the interval between a musical pitch and another pitch at half or double its frequency, which means the octave is proportional to power of 2 . The human brain, however, perceive the same difference when an octave changes higher or lower. For example, when an 88-key piano played the first $\mathrm{C}\left(\mathrm{C}_{1}\right.$, $32.70 \mathrm{~Hz})$ to the last $\mathrm{C}\left(\mathrm{C}_{8}, 4186.01 \mathrm{~Hz}\right)[3]$, the frequency of the last key is $2^{7}$ times more than that of the first key because of the seven octave difference. The human brain, however, recognize that $C_{8}$ is 7 times higher pitch than $C_{1}$. Every interval between keys are the same for human ear, whereas frequency differences between keys are different. The equation is as below:

$$
f(n)=440 \times 2^{(n-49) / 12} \mathrm{~Hz}
$$

where $f$ is the audio frequency and $n$ is the number of the key. The forty-ninth piano key (major $\mathrm{A}_{4}$, concert pitch) is $440 \mathrm{~Hz}$ and one octave has twelve keys. From the Eq. (7), major $\mathrm{A}_{5}$ (the sixty-first key) is $880 \mathrm{~Hz}$, major $\mathrm{A}_{3}$ (the thirty-seventh key) is 220 $\mathrm{Hz}$, and major $\mathrm{C}_{4}$ (the fortieth key, the middle $\mathrm{C}$ ) is $261.63 \mathrm{~Hz}$.

When digital device generate sound with a single frequency, it sounds like a beep. Depending on the types of waveform (e.g. sine, square, sawtooth), different beep sounds are generated. Unlike sound with a single frequency, natural sound occurs with combinations of different waveforms and frequencies. All audio waveforms can be expressed by sine and cosine equations, which is the principle of the Fourier analysis. When most of the musical instruments are played with a fundamental frequency $f$, sound naturally contains multiple frequency components, such as $2 f, 3 f, 4 f, 5 f$, and more (called overtones or harmonic partials). According to the nature of the harmonic feature, the perfect octave is defined as an interval between $f$ and $2 f$. As harmonic intervals, the perfect fifth ( $2 f$ and $3 f)$ and the perfect fourth ( $3 f$ and $4 f)$ are also defined in music. The combinations with harmonic partials can generate a harmony which human ear hear pleasantly. When two or more auditory feedbacks are generated, it would be better to choose the base frequencies with harmonic intervals. When start frequency is different, the range of frequency should be different for the same feedback effect.

\subsection{Measurement of Acoustic Parameters}

This subsection is to discuss the measurement of decibel and pitch. Before applied to auditory feedback, auditory parameters should be measured behind the audio source and before the terminal receptor-the ear of the listener. A decibel meter can measure the loudness, and spectral analysis of sound can describe the distribution of audio frequencies, supporting the estimation of pitch. If there exists a certain distance between 
a sound source and a listener, the physical sound is affected by the environmental temperature, the air reference pressure, and the humidity of the air. To achieve a reliable result, a microphone can be used to measure the given amplitude and frequency of the acoustic event at a certain location. Discrete-time Fourier transform (DTFT) can be used to analyze the received waveform and harmonic partials in the frequency domain (Oppenheim \& Schafer, 1999). With the use of headphones impact of such environmental factors can be reduced.

\subsection{Motion Capture: The Measurement of Movement Parameters}

If the auditory information should be based on movement parameters directly, a digital motion capture system can be used to measure movement parameters. For the collection of kinematic movement data, there exists three methods of motion capture: optical, non-optical and marker-less methods. Optical methods implement cameras, markers and optic sources such as visible light and infrared ray (IR) (Kirk, O'Brien, \& Forsyth, 2005). Markers on actor's special suit reflect optical wave from the optic source, and then cameras record movement of markers. From several numbers of cameras, information of marker positions is collected, and human movement is regenerated in 3-D space by a special rendering software. Although management of hidden markers (Silaghi, Plänkers, Boulic, Fua \& Thalmann, 1998) and a high cost of multiple cameras can be problematic, optical motion capture has been matured with entertainment and computer game industry.

Non-optical methods have been developed with mechanical sensors, magnetic marker systems, and inertial sensors (Brodie, Walmsley \& Page, 2008). Mechanical methods implements banding angles of wires on human joints, whereas magnetic marker systems measure human movement considering the nearby magnetic field. The inertial sensors (accelerometers, gyroscopes) measure acceleration and angular velocity of body segments as a base for computing movement parameters (relative velocity, position) and finally, human motions and postures. Nevertheless, disadvantages of the technology have been reported. Inertial sensor systems suffer from error accumulation resulted from double integration of acceleration, which provokes position drift effects. Body mounted mechanical sensor systems can affect human motion and limit the range of measurement. Magnetic sensor systems are interfered by magnetic distortion from the ambient metal and electric field.

With cutting-edge image processing technology, marker-less systems are developed, which animates human motions with vision (Moeslund, Hilton \& Krüger, 2006). Dual camera systems can estimate image depth information. The disadvantage of the technique arelimitations in terms of hidden areas behind body parts or used objects. Simple markerless systems, therefore, cannot compute the human model in full 3-D space, so it is called 2.5-D motion capture. The dual camera solutions, however, can guarantee simplicity. This makes such technology applicable at home and popular in the computer game industry. 
On the one hand, studies on enhancing accuracy and preciseness of IMU were conducted. Fusion of above mentioned technologies have been recently established, compensating for the disadvantages. For example, an inertial measurement units (IMU) includes not only inertial sensors (accelerometer, gyroscope), but also magnetometers (Rios \& White, 2002), global positioning systems (GPS) (Hellmers, Norrdine, Blankenbach \& Eichhorn, 2003), or cameras (Hesch, Kottas, Bowman \& Roumeliotis, 2014). With combined processing of the data, drift effects of inertial sensors can be reduced. Fusion technology has quickly grown in human kinematic analysis as well as in entertainment-, education- and sports-industry. On the other hand, researchers have focused on reducing the number of sensors. Depending on the particular purpose, one or more parts of the body can be measured: the lower body (Rueterbories, Spaich, Larsen, \& Andersen, 2010), the upper body (Cromwell \& Wellmon, 2001), the ear (Atallah, Aziz, Lo \& Yang, 2009), and the head (Hwang, Reh, Effenberg \& Blume, 2018). For the generation of auditory movement information, different parts of the body have been tracked, as the feet (Reh, Hwang, Michalkev \& Effenberg, 2016), or the arms (Schmitz, Bergmann, Effenberg, Krewer, Hwang, \& Mueller, 2018). Additionally, objects handled by humans have been sonified, as recently realized on a joint task performed witha tablet PC (Hwang, Schmitz, Klemmt, Brinkop, Ghai \& Stoica et al. 2018).

In real-time auditory feedback of movement, it should take less than $100 \mathrm{~ms}$ from the motion capture to sound generation (Stein \& Meredith, 1993) to achieve an integration of the auditory information with current perceptual information of other modality. In this context, IMUs are suitable for real-time movement feedback because the latency from a sensor to a host PC takes only about $30 \mathrm{~ms}$.

\subsection{Connecting Motion Capture With Audio Systems}

This subsection is to inform about different types of interfaces between motion capture systems and audio systems, and also about the reasons of the latency of data transmission. In a motion capture system, kinematic data can be transmitted to an audio system by wired or wireless connections. When two systems or software solutions are installed on a single computer, communication between two processes can be realized through local host IP address. In this case, the delay can be ignored because both system components are internally wired, which causes only minimal delay. If the required data format (osc, json, MIDI) of the audio software is different from the data-format of the motion capture software, a managing program is needed.

When the audio system and the motion capture system are connected with an external wire, a common hardware interface is needed, such as USB or AV-cable. Concerning a common data format, a number of digital instruments communicate using the "Musical Instruments Digital Interface-Standard" (MIDI), avoiding data transmission distortion or any serious delay. For a wireless connection between two systems, the connection can be established with WiFi or Bluetooth - with a certain risk of data congestion and data loss. The audio data should be created in a common format like osc, 
json, or MIDI. Nowadays, a PC can be used as an audio system with free software or library, such as CSound, pureData, and a MIDI generator.

\subsection{Noise Reduction}

This subsection should highlight the importance of noise reduction for the creation of high quality sound. In the sonification system, noise reduction is very important because it might distort feedback effects on listeners and impact the final results. It can make listeners tired or unpleased, even up to damages of the hearing ability. To ensure a pleasant hearing experience, the maximum amplitude of sound should be limited with a proper threshold value. For preciseness of feedback, higher bit-depth is beneficial for the reduction of quantization errors, by increasing the signal-to-noise ratio (SNR) (Bialkowski, 1988). In addition, a finite impulse response (FIR) digital filter aids to makes the signal smooth and to reduce noise from hardware or software systems. A digital filter can compensate for dropped sample points during wireless transmission. In conclusion, noise is reduced by higher bit-depth, higher sampling ratio, and FIR filter. In addition, sound engineer should care about the hardware noise and environmental acoustic noise.

\subsection{Applications}

This subsection introduces example applications that implement artificial auditory information in various situations. Real-time kinematic auditory feedback has been recently applied to enhance motor control and learning (Effenberg, 2005; Effenberg et al., 2016). Additional real-time auditory feedback of movement also improves tempo and preciseness of motor learning (Effenberg et al., 2016). If kinematic real-time acoustics is integrated with other current perceptual information, it can support the development of sensorimotor representations obviously without conscious attention, as indicated by Effenberg, 2005, Effenberg, Fehse et al. 2016. Furthermore, auditory feedback can be divided into performance-based auditory feedback (PAF) and effect-based auditory feedback (EAF). Additional PAF supports the quality of movement, whereas the EAF indicates the result of movement (Magill \& Anderson, 2007; Schmidt \& Wrisberg, 2008). It is reported that PAF (Weeks \& Kordus, 1998; Nunes, Souza, Basso, Monteiro, Corrêa \& Santos, 2014; Sharma, Chevidikunnan, Khan \& Gaowgzeh, 2016) and EAF (Schmidt \& Wrisberg, 2008; Sharma et al., 2016; Winstein, 1991) have beneficial effects on motor learning under different conditions. Artificial real-time auditory feedback can be applied to post-hospital gait rehabilitation (Reh et al., 2016), and can enhance proprioceptive repositioning accuracy of knee-angles (Ghai et al. 2018). Above that, also the visual perception of gross-motor movement frequencies can be tuned systematically by concordant kinematic sonification in terms of acceleration or deceleration (Effenberg \& Schmitz, 2018). Those types of auditory feedback can also contribute to interpersonal coordination (Schmitz \& Effenberg, 2017, Hwang \& Schmitz et al. 2018). Further in future, we might be even able to support human-robot interactions by adding an acoustic profile to robot kinematics to address the action-observation system, as already indicated on a human avatar by Schmitz \& Mohammadi et al., 2013. 


\section{Conclusion}

The present article describes the potential of artificially generated auditory movement information on perception and action. From different perspectives we scrutinize how auditory information addresses multisensory percepts of complex movements as well as relatively simple movements. Both frameworks refer to the internal model theory, but differ with respect to their focus: Adding artificial movement acoustics to motion perception allows to shape multisensory movement representations in order to learn, relearn or maintain movement competences (section 1). Here, the key mechanisms refer to the shaping of forward models by sound for the initial learning phases. Forward models represent the prediction of sensory/perceptual consequences of actions, which are integrated with perceptual information from different sensory modalities and thereby affect overall motion percepts. The framework described in section 2 predominantly explains how perceptual information is linked to internal models and how effectors can access this information. Key elements are the coupling of sensory modalities and effectors to internal models by default and the emergence of weighting and switch functions through training: Setting of weights and switches determines whether adaptation of internal models is limited to the trained task or whether it generalizes to novel situations and movements.

Whereas these behavioral frameworks refer to the mechanisms of feedback, section 3 highlights the effects of external auditory information on movement competences from neurologic and neuroscientific perspectives. In this section, an emphasis has been laid to suggest the influence of rhythmic auditory cueing on cyclic tasks, for instance, gait. Here, the rhythmic auditory cueing, which is adapted according to the patients' preferred cadence, might allow the development of stable feed-forward predictive models, thereby resulting in an efficient motor planning, and execution. The section concludes that providing external auditory cueing supports the development of perceptuomotor representations, facilitating plasticity and reducing cognitive-motor overload for supporting motor recovery. The section also explains possible neurophysiological mechanisms which might underlie this effect.

Finally, section 4 describes a framework for the generation of movement sounds from the perspective of an engineer. There exists a strong relationship between human perception and the physics of sound. Compared to most mechanical and electrical systems the human physics is characterized by a huge number of degrees of freedom which have to be controlled by a huge number of muscles, being innervated in an adequate way. Even with reference to the internal model-theory this is a very challenging task from the view of an engineer. Future research on computational models of the proposed internal models should be usable as a base also for research about new kinds of efficiency enhanced feedback in the auditory domain.

Acknowledgments. This research was supported by European Commission H2020-FETPROACT-2014 No. 641321 


\section{References}

1. Asanuma, H., \& Keller, A. (1991). Neuronal mechanisms of motor learning in mammals. Neuroreport, 2(5), 217-224.

2. Atallah, L., Aziz, O., Lo, B., \& Yang, G. Z. (2009, June). Detecting walking gait impairment with an ear-worn sensor. In Wearable and Implantable Body Sensor Networks, 2009. BSN 2009. Sixth International Workshop on (pp. 175-180). IEEE.

3. Bastian, A. J. (2008). Understanding sensorimotor adaptation and learning for rehabilitation. Current Opinion in Neurology, 21(6), 628-633.

4. Baumann, O., \& M.W. Greenlee. 2006. Neural correlates of coherent audiovisual motion perception. Cerebral Cortex. 17: 1433-1443.

5. Bernier, P., Gauthier, G., \& Blouin, J. (2007). Evidence for distinct, differentially adaptable sensorimotor transformations for reaches to visual and proprioceptive targets. Journal of Neurophysiology, 98, 1815-1819.

6. Berthoz, A., \& I. Viaud-Delmon (1999). Multisensory integration in spatial orientation. Current opinion in Neurobiology 9(6): 708-712.

7. Bialkowski, S. E. (1988). Real-time digital filters: infinite impulse response filters. Analytical Chemistry, 60(6), 403A-413A.

8. Bishop, L., \& Goebl, W. (2018). Beating time: How ensemble musicians' cueing gestures communicate beat position and tempo. Psychology of Music, 46(1), 84-106. DOI: $10.1177 / 0305735617702971$.

9. Bock, O. (2013). Basic principles of sensorimotor adaptation to different distortions with different effectors and movement types: a review and synthesis of behavioral findings. Frontiers in Human Neuroscience, 7: 81.

10. Bock, O., \& Schmitz, G. (2013). Transfer of visuomotor adaptation to unpractised hands and sensory modalities. Psychology, 4(12), 1004-1007. DOI:10.4236/psych.2013.412145.

11. Bock, O., Schmitz, G., \& Grigorova, V. (2008). Transfer of adaptation between ocular saccades and arm movements. Human Movement Science, 27, 383-395.

12. Brodie, M., Walmsley, A., \& Page, W. (2008). Fusion motion capture: a prototype system using inertial measurement units and GPS for the biomechanical analysis of ski racing. Sports Technology, 1(1), 17-28.

13. Buchecker, M., Wegenkittl, S., Stöggl, T., \& Müller, E. (2017). Unstable Footwear Affects Magnitude and Structure of Variability in Postural Control. Motor control, 1-35.

14. Calvert, G. A., Spence, C., \& Stein, B. E. (Eds.). (2004). The handbook of multisensory processes. Cambridge, Massachusetts: MIT Press.

15. Campos-Sousa, I. S., Campos-Sousa, R. N., Ataide, L., Jr., Soares, M. M., \& Almeida, K. J. (2010). Executive dysfunction and motor symptoms in Parkinson's disease. Arquivos de NeuroPsiquiatria, 68(2), 246-251.

16. Chen, L., \& Vroomen, Y. (2013). Intersensory binding across space and time: a tutorial review. Attention, Perception, \& Psychophysics, 75, 790-811.

17. Cohen, M. (1974). Changes in auditory localization following prismatic exposure under continuous and terminal visual feedback. Perceptual and Motor Skills, 38, 1202.

18. Craske, B. (1966). Intermodal transfer of adaptation to displacement. Nature, 5037, 765.

19. Cromwell, R., \& Wellmon, R. (2001). Sagittal plane head stabilization during level walking and ambulation on stairs. Physiotherapy Research International, 6(3), 179-192.

20. Cunnington, R., Iansek, R., Bradshaw, J. L., \& Phillips, J. G. (1995). Movement-related potentials in Parkinson's disease. Brain, 118(4), 935-950. 
21. D’Ausilio, A., Badino, L., Li, Y., Tokay, S., Craighero, L., Canto, R., Aloimonos, Y., \& Fadiga, L. (2012). Leadership in orchestra emerges from the causal relationships of movement kinematics. PLoS ONE, 7(5), e35757.

22. D'Ausilio, A., Novembre, G., Fadiga, L., \& Keller, P. E. (2015). What can music tell us about social interaction? Trends in Cognitive Sciences, 19(3), 111-114.

23. Debaere, F., Wenderoth, N., Sunaert, S., Van Hecke, P., \& Swinnen, S. P. (2003). Internal vs external generation of movements: differential neural pathways involved in bimanual coordination performed in the presence or absence of augmented visual feedback. Neuroimage, 19(3), 764-776.

24. Delignières, D., \& Torre, K. (2009). Fractal dynamics of human gait: a reassessment of the 1996 data of Hausdorff et al. Journal of Applied Physiology, 106(4), 1272-1279.

25. Demos, A. P., Chaffin, R., Begosh, K. T., Daniels, J. R., \& Marsh, K. L. (2012). Rocking to the beat: Effects of music and partner's movements on spontaneous interpersonal coordination. Journal of Experimental Psychology: General, 141(1), 49.

26. Dijkerman, H.C., McIntosh, R. D., Anema, H. A., de Haan, E. H., Kappelle, L. J., \& Milner, A. D. (2006). Reaching errors in optic ataxia are linked to eye position rather than head or body position. Neuropsychologia, 44(13), 2766-2773.

27. Dubus, G., \& Bresin, R. (2013). A systematic review of mapping strategies for the sonification of physical quantities. PloS one, 8(12), e82491.

28. Effenberg, A. O. (2005). Movement sonification: Effects on perception and action. IEEE multimedia, 12(2), 53-59.

29. Effenberg, A. O., Fehse, U., Schmitz, G., Krueger, B., \& Mechling, H. (2016). Movement sonification: Effects on motor learning beyond rhythmic adjustments. Frontiers in Neuroscience, 10. DOI:10.3389/fnins.2016.00219.

30. Effenberg, A. O., Schmitz, G., Baumann, F., Rosenhahn, B., \& Kroeger, D. (2015). SoundScript-Supporting the Acquisition of Character Writing by Multisensory Integration. Open Psychology Journal, 8(3), 230-237. DOI:10.2174/1874350101508010230.

31. Effenberg, A.O., \& Schmitz, G. (2018). Acceleration and deceleration at constant speed: systematic modulation of motion perception by kinematic sonification. Annals of the New York Academy of Sciences. DOI:10.1111/nyas.13693.

32. El-Tamawy, M. S., Darwish, M. H., \& Khallaf, M. E. (2012). Effects of augmented proprioceptive cues on the parameters of gait of individuals with Parkinson's disease. Annals of Indian Academy of Neurology, 15(4), 267-272.

33. Felix, R. A., Fridberger, A., Leijon, S., Berrebi, A. S., \& Magnusson, A. K. (2011). Sound rhythms are encoded by postinhibitory rebound spiking in the superior paraolivary nucleus. Journal of Neuroscience, 31(35), 12566-12578.

34. Flannigan, J. C., Posthuma, R. J., Lombardo, J. N., Murray, C. \& Cressmann, E. K. (2018). Adaptation to proprioceptive targets following visuomotor adaptation. Experimental Brain Research, 236, 419-432.

35. Ford, M. P., Malone, L. A., Nyikos, I., Yelisetty, R., \& Bickel, C. S. (2010). Gait training with progressive external auditory cueing in persons with Parkinson's disease. Archives of Physical Medicine and Rehabilitation, 91(8), 1255-1261.

36. Fujioka, T., Trainor, L. J., Large, E. W., \& Ross, B. (2012). Internalized timing of isochronous sounds is represented in neuromagnetic beta oscillations. Journal of Neuroscience, 32(5), 17911802.

37. Galea, J., \& Miall, R. (2006). Concurrent adaptation to opposing visual displacements during an alternating movement. Experimental Brain Research, 175, 676-688.

38. Ghai, S., Ghai, I., \& Effenberg, A. O. (2017). Effect of rhythmic auditory cueing on aging gait: a systematic review and meta-analysis. Aging and Disease, 131-200. 
39. Ghai, S., Ghai, I., \& Effenberg, A. O. (2017). Effects of dual-task training and dual-tasks on postural stability: A systematic review and meta-analysis. Clinical Interventions in Aging, 12, 557-577.

40. Ghai, S., Ghai, I., \& Effenberg, A. O. (2018). Effect of rhythmic auditory cueing on gait in cerebral palsy: a systematic review and meta-analysis. Neuropsychiatric Disease and Treatment, 14, 43-59.

41. Ghai, S., Ghai, I., Schmitz, G., \& Effenberg, A. O. (2018). Effect of rhythmic auditory cueing on parkinsonian gait: A systematic review and meta-analysis. Scientific Reports, 8(1), 506.

42. Ghai, S., Schmitz, G., Hwang, T.-H., \& Effenberg, A. O. (2018). Auditory Proprioceptive Integration: Effects of Real-Time Kinematic Auditory Feedback on Knee Proprioception. Frontiers in Neuroscience, 12(142).

43. Gibson, J. J. (1966) The senses considered as perceptual systems. Houghton-Mifflin.

44. Gibson, J. J. (1979) The ecological approach to visual perception. Houghton-Mifflin.

45. Goodman, J. R., Isenhower, R. W., Marsh, K., Schmidt, R., \& Richardson, M. (2005). The interpersonal phase entrainment of rocking chair movements. Studies in Perception and Action VIII: Thirteenth International Conference on Perception and Action (Heft, H. \& Marsh, KL, eds).

46. Haar, S., Donchin, O., \& Dinstein, I. (2015). Dissociating visual and motor directional selectivity using visuomotor adaptation. Journal of Neuroscience, 35(17), 6813-6821.

47. Hameed, S., Ferris, T., Jayaraman, S., \& Sarter, N. (2009). Using informative peripheral visual and tactile cues to support task and interruption management. Human Factors, 51(2), 126-135.

48. Harris, C. S. (1963). Adaptation to displaced vision: visual, motor, or proprioceptive change? Science, 140, 812-813.

49. Harris, C. S. (1965). Perceptual adaptation to inverted, reversed, and displaced vision. Psychological Review, 72(6), 419-444.

50. Hatada, Y., Miall, R. C., \& Rossetti, Y. (2006). Two waves of a long-lasting aftereffect of prism adaptation measured over 7 days. Experimental Brain Research, 169(3), 417-426.

51. Hausdorff, J. M., Lowenthal, J., Herman, T., Gruendlinger, L., Peretz, C., \& Giladi, N. (2007). Rhythmic auditory stimulation modulates gait variability in Parkinson's disease. European Journal of Neuroscience, 26(8), 2369-2375.

52. Hausdorff, J. M., Purdon, P. L., Peng, C., Ladin, Z., Wei, J. Y., \& Goldberger, A. L. (1996). Fractal dynamics of human gait: stability of long-range correlations in stride interval fluctuations. Journal of Applied Physiology, 80(5), 1448-1457.

53. Hellmers, H., Norrdine, A., Blankenbach, J., \& Eichhorn, A. (2013, October). An IMU/magnetometer-based indoor positioning system using Kalman filtering. In Indoor Positioning and Indoor Navigation (IPIN), 2013 International Conference on (pp. 1-9). IEEE.

54. Hesch, J. A., Kottas, D. G., Bowman, S. L., \& Roumeliotis, S. I. (2014). Camera-IMU-based localization: Observability analysis and consistency improvement. The International Journal of Robotics Research, 33(1), 182-201.

55. Hopkins, K., Kass, S. J., Blalock, L. D., \& Brill, J. C. (2017). Effectiveness of auditory and tactile crossmodal cues in a dual-task visual and auditory scenario. Ergonomics, 60(5), 692700 .

56. Hove, M. J., Suzuki, K., Uchitomi, H., Orimo, S., \& Miyake, Y. (2012). Interactive rhythmic auditory stimulation reinstates natural $1 / \mathrm{f}$ timing in gait of Parkinson's patients. PloS one, 7(3), e32600.

57. Hwang, T. H., Reh, J., Effenberg, A. O., \& Blume, H. (2018). Real-time gait analysis using a single head-worn inertial measurement unit. IEEE Transactions on Consumer Electronics, 64(2). DOI:10.1109/TCE.2018.2843289. 
58. Hwang, T.-H., Schmitz, G., Klemmt, K., Brinkop, L., Ghai, S., Stoica, M. et al., (2018). Effect and performance-based auditory feedback on interpersonal coordination. Frontiers in Psychology 9:404. DOI:10.3389/fpsyg.2018.00404.

59. Imamizu, H., \& Shimojo, S. (1995). The locus of visual-motor learning at the task or manipulator level: Implications from intermanual transfer. Journal of Experimental Psychology: Human Perception and Performance, 21, 719-733.

60. Imamizu, H., Sugimoto, N., Osu, R., Tsutsui, K., Sugiyama, K., Wada, Y., \& Kawato, M. (2007). Explicit contextual information selectively contributes to predictive switching of internal models. Experimental Brain Research, 181(3), 395-408.

61. Johansson, B. B. (2012). Multisensory stimulation in stroke rehabilitation. Frontiers in Human Neuroscience 6: 60 .

62. Kagerer, F. A., \& Contreras-Vidal, J. L. (2009). Adaptation of sound localization induced by rotated visual feedback in reaching movements. Experimental Brain Research, 193(2), 315321.

63. Keller, P. E., Knoblich, G., \& Repp, B. H. (2007). Pianists duet better when they play with themselves: On the possible role of action simulation in synchronization. Consciousness and cognition, 16(1), 102-111.

64. Keller, P. E., Novembre, G., \& Hove, M. J. (2014). Rhythm in joint action: psychological and neurophysiological mechanisms for real-time interpersonal coordination. Philosophical Transactions of the Royal Society B: Biological Sciences, 369(1658), 20130394.

65. Keysers, C., Kohler, E., Umilta, M. A., Nanetti, L., Fogassi, L., \& Gallese, V. (2003). Audiovisual mirror neurons and action recognition. Experimental Brain Research(153), 628-636.

66. Khoramshahi M., Shukla A., Raffard S., Bardy B. G., \& Billard A. (2016). Role of Gaze Cues in Interpersonal Motor Coordination: Towards Higher Affiliation in Human-Robot Interaction. PLoS ONE 11(6): e0156874. DOI:10.1371/journal.pone.0156874.

67. Kirk, A. G., O'Brien, J. F., \& Forsyth, D. A. (2005, June). Skeletal parameter estimation from optical motion capture data. In Computer Vision and Pattern Recognition, 2005. CVPR 2005. IEEE Computer Society Conference on (Vol. 2, pp. 782-788). IEEE.

68. Knoblich, G., Butterfill, S., \& Sebanz, N. (2011). 3 Psychological research on joint action: theory and data. Psychology of Learning and Motivation-Advances in Research and Theory, $54,59$.

69. Kohler, E., Keysers, C., Umilta, M. A., Fogassi, L., Gallese, V., \& Rizzolati, G. (2002). Hearing sounds, understanding actions: action representation in mirror neurons. Science(297), 846-848.

70. Kohler, I. (1951). Über Aufbau und Wandlungen der Wahrnehmungswelt, insbesondere über bedingte Empfindungen.': In Kommission bei RM Rohrer.

71. Kohler, I. (1963). The formation and transformation of the perceptual world. Psychological issues.

72. Lacquaniti, F., Bosco, G., Gravano, S., Indovina, I., La Scaleia, B., Maffei, V., et al. (2014). Multisensory integration and internal models for sensing gravity effects in primates. BioMed research international. 2014.

73. Lahav, A., Saltzman, E., \& Schlaug, G. (2007). Action Representation of Sound: Audiomotor Recognition Network While Listening to Newly Acquired Actions. The Journal of Neuroscience, 27(2), 308-314.

74. Lee, J. Y., \& Schweighofer, N. (2009). Dual adaptation supports a parallel architecture of motor memory. Journal of Neuroscience, 29(33), 10396-10404.

75. Lewald, J.m \& Getzmann, S. (2006). Horizontal and vertical effects of eye-position on sound localization. Hearing Research, 213, 99-106.

76. Lohnes, C. A., \& Earhart, G. M. (2011). The impact of attentional, auditory, and combined cues on walking during single and cognitive dual tasks in Parkinson disease. Gait and Posture, 33(3), 478-483. 
77. Magescas, F., \& Prablanc, C. (2006). Automatic drive of limb motor plasticity. Journal of Cognitive Neuroscience, 18(1), 75-83.

78. Magill, R. A., \& Anderson, D. I. (2007). Motor learning and control: Concepts and applications (Vol. 11). New York: McGraw-Hill.

79. Martin, L. M., \& Newman, C. V. (1980). Simultaneous right- and left-hand adaptation in opposite lateral directions following bidirectional optical displacement. Bulletin of the Psychonomic Society, 16(6), 432-434.

80. Michel, C., Pisella, L., Prablanc, C., Rode, G., \& Rossetti, Y. (2007). Enhancing visuomotor adaptation by reducing error signals: Single-step (Aware) versus multiplestep (Unaware) exposure to wedge prisms. Journal of Cognitive Neuroscience, 19(2), 341-350.

81. Mikaelian, H. (1972). Lack of bilateral generalization of adaptation to auditory rearrangement. Perception \& Psychophysics, 11(3), 222-224.

82. Mikaelian, H. (1974). Adaptation to displaced hearing: A nonproprioceptive change. Journal of Experimental Psychology, 103, 326-330.

83. Miyata, K., Varlet, M., Miura, A., Kudo, K., \& Keller, P. E. (2017). Modulation of individual auditory-motor coordination dynamics through interpersonal visual coupling. Scientific Reports, 7, 16220. DOI:10.1038/s41598-017-16151-5.

84. Moeslund, T. B., Hilton, A., \& Krueger, V. (2006). A survey of advances in vision-based human motion capture and analysis. Computer vision and image understanding, 104(2-3), 90-126.

85. Morton, S. M., \& Bastian, A. J. (2004). Prism Adaptation During Walking Generalizes to Reaching and Requires the Cerebellum. The Journal of Neuroscience, 92: 2497-2509.

86. Mueller, G., \& Moeser, M. (Eds.). (2012). Handbook of engineering acoustics. Springer Science and Business Media.

87. Murgia, M., Santoro, I., Tamburini, G., Prpic, V., Sors, F., Galmonte, A., \& Agostini, T. (2016). Ecological sounds affect breath duration more than artificial sounds. Psychological Research, 80(1), 76-81.

88. Nieuwboer, A., Baker, K., Willems, A.-M., Jones, D., Spildooren, J., Lim, et al., (2009). The short-term effects of different cueing modalities on turn speed in people with Parkinson's disease. Neurorehabilitation and Neural Repair.

89. Nieuwboer, A., Kwakkel, G., Rochester, L., Jones, D., van Wegen, E., Willems, A. M., et al., (2007). Cueing training in the home improves gait-related mobility in Parkinson's disease: the RESCUE trial. Journal of Neurology, Neurosurgery and Psychiatry, 78(2), 134-140.

90. Nombela, C., Hughes, L. E., Owen, A. M., \& Grahn, J. A. (2013). Into the groove: can rhythm influence Parkinson's disease? Neuroscience and Biobehavioral Reviews, 37(10), 2564-2570.

91. Nunes, M. E., Souza, M. G., Basso, L., Monteiro, C., Corrêa, U. C., \& Santos, S. (2014). Frequency of provision of knowledge of performance on skill acquisition in older persons. Frontiers in psychology, 5, 1454.

92. Oostwoud Wijdenes, L., \& Medendorp,W. P. (2017). State Estimation for Early Feedback Responses in Reaching: Intramodal or Multimodal? Frontiers in Integrative Neuroscience, 11:38. DOI:10.3389/fnint.2017.00038.

93. Oppenheim, A. V. (1999). Discrete-time signal processing. Pearson Education India.

94. Oscari, F., Secoli, R., Avanzini, F., Rosati, G., \& Reinkensmeyer, D. J. (2012). Substituting auditory for visual feedback to adapt to altered dynamic and kinematic environments during reaching. Experimental Brain Research, 221, 33-41.

95. Oullier, O., De Guzman, G. C., Jantzen, K. J., Lagarde, J., \& Scott Kelso, J. (2008). Social coordination dynamics: Measuring human bonding. Social neuroscience, 3(2), 178-192.

96. Prablanc, C., Tzavaras, A., \& Jeannerod, M. (1975). Adaptation of the two arms to opposite prism displacements. The Quarterly Journal of Experimental Psychology, 27(4), 667-671. 
97. Rinehart, N. J., Bellgrove, M. A., Tonge, B. J., Brereton, A. V., Howells-Rankin, D., \& Bradshaw, J. L. (2006). An examination of movement kinematics in young people with high-functioning autism and Asperger's disorder: further evidence for a motor planning deficit. Journal of Autism and Developmental Disorders, 36(6), 757-767.

98. Reh, J., Hwang, T. H., Michalke, V., \& Effenberg, A. O (2016, September). Instruction and real-time sonification for gait rehabilitation after unilateral hip arthroplasty. In 11th Joint Conference on Motor Control Learning Biomechanics Training (pp. 1-2), DVS.

99. Rios, J. A., \& White, E. (2002). Fusion filter algorithm enhancements for a MEMS GPS/IMU. Crossbow Technology, Inc, 1-12.

100. Rueterbories, J., Spaich, E. G., Larsen, B., \& Andersen, O. K. (2010). Methods for gait event detection and analysis in ambulatory systems. Medical Engineering and Physics, 32(6), 545552.

101. Sainburg, R., \& Wang, J. (2002). Interlimb transfer of visuomotor rotations: independence of direction and final position information. Experimental Brain Research, 145, 437-447.

102. Schaefer, R. S. 2014. Auditory rhythmic cueing in movement rehabilitation: findings and possible mechanisms. Philosophical Transactions of the Royal Society B: Biological Sciences. 369: 20130402.

103. Schmitz, G. \& Bock, O. (2017). Properties of intermodal transfer after dual visuo- and auditorymotor adaptation. Human Movement Science, 55, 108-120.

104. Schmitz, G. (2014). Visuo- und audiomotorische Adaptation. Schorndorf: Hofmann-Verlag. ISBN 978-3-7780-4850-4.

105. Schmitz, G. (2016). Interference between adaptation to double steps and adaptation to rotated feedback in spite of differences in directional selectivity. Experimental Brain Research, 234, 1491-1504. DOI:10.1007/s00221-016-4559-y.

106. Schmitz, G., \& Effenberg, A. O. (2016). Sound Joint Actions in Rowing and Swimming. Moving Bodies in Interaction - Interacting Bodies in Motion. C. Meyer and U. v. Wedelstaedt. Amsterdam, John Benjamins Publishing Company.

107. Schmitz, G., Bergmann, J., Effenberg, A. O., Krewer, C., Hwang, T. H., \& Mueller, F. (2018). Movement sonification in stroke rehabilition. Frontiers in Neurology, 9, 389.

108. Schmitz, G., \& Bock, O. (2014). A comparison of sensorimotor adaptation in the visual and in the auditory modality. PLoS One, 9(9), e107834.

109. Schmitz, G., \& Effenberg, A. O. (2012). Perceptual effects of auditory information about own and other movements. 18th International Conference on Auditory Display, Atlanta, GA, USA.

110. Schmitz, G., \& Effenberg, A. O. (2017). Schlagmann 2.0 - Bewegungsakustische Dimensionen interpersonaler Koordination im Mannschaftssport. German Journal of Exercise and Sport Research, 47(3), 232-245.

111. Schmitz, G., \& Grigorova, V. (2017). Alternating adaptation of eye and hand movements to opposite directed double steps. Journal of Motor Behavior, 49(3), 255-264. DOI:10.1080/00222895.2016.1191419

112. Schmitz, G., Kroeger, D., \& Effenberg, A. O. (2014). A mobile sonification system for stroke rehabilitation. The 20th International Conference on Auditory Display, New York.

113. Schmitz, G., Mohammadi, B., Hammer, A., Heldmann, M., Samii, A., Münte, T. F., \& Effenberg, A. O. (2013). Observation of sonified movements engages a basal ganglia frontocortical network. BMC Neuroscience, 14(32). DOI:10.1186/1471-2202-14-32.

114. Sebanz, N., \& G. Knoblich (2009). Prediction in joint action: What, when, and where. Topics in Cognitive Science 1(2): 353-367.

115. Sebanz, N., Bekkering, H., \& Knoblich, G. (2006). Joint action: bodies and minds moving together. Trends in Cognitive Sciences, 10(2), 70-76.

116. Seitz, A. R., Kim, R., \& Shams, L. (2006). Sound facilitates visual learning. Current Biology, 16(14), 1422 - 1427

117. Sengpielaudio hompage: http://www.sengpielaudio.com/calculator-notenames.htm. 
118. Shadmehr, R., Smith, R. A., \& Krakauer, J. W. (2010). Error correction, sensory prediction, and adaptation in motor control. Annual Reviews of Neuroscience, 33, 89-108.

119. Shams, L.; \& Seitz, A. R. (2008). Benefits of multisensory learning. Trends in Cognitive Science 12(11): 411-417.

120. Sharma, D. A., Chevidikunnan, M. F., Khan, F. R., \& Gaowgzeh, R. A. (2016). Effectiveness of knowledge of result and knowledge of performance in the learning of a skilled motor activity by healthy young adults. Journal of physical therapy science, 28(5), 1482-1486.

121. Silaghi, M. C., Plaenkers, R., Boulic, R., Fua, P., \& Thalmann, D. (1998). Local and global skeleton fitting techniques for optical motion capture. In Modelling and Motion Capture Techniques for Virtual Environments (pp. 26-40). Springer, Berlin, Heidelberg.

122. Smith, M. A., Ghazizadeh, A., \& Shadmehr, R. (2006). Interacting Adaptive Processes with Different Timescales Underlie Short-Term Motor Learning. PLoS Biology, e179. DOI:10.1371/journal.pbio.0040179.

123. Spence, C. (2015). Coss-modal perceptual organization. The Oxford handbook of percepttual organization. J. Wagemans. Oxford, UK, Oxford University Press.

124. Spence, C., \& Driver, J. (Eds.) (2004). Crossmodal space and crossmodal attention. Oxford, Oxford University Press.

125. Stein, B. E., \& Meredith, M. A. (1993). The Merging of the Senses. Cambridge, MIT Press.

126. Stein, B. E., \& Stanford, T. R. (2008). Multisensory integration: current issues from the perspective of the single neuron. Nature Reviews Neuroscience 9(4): 255-266.

127. Stoffregen, T.A. \& Bardy, B.G. (2001). On specification and the senses. Behavioral and Brain Sciences 24: 195-213; discussion 213-161.

128. Suh, J. H., Han, S. J., Jeon, S. Y., Kim, H. J., Lee, J. E., Yoon, T. S., \& Chong, H. J. (2014). Effect of rhythmic auditory stimulation on gait and balance in hemiplegic stroke patients. NeuroRehabilitation, 34(1), 193-199.

129. Tagliabue, M., \& McIntyre, J. (2014). A modular theory of multisensory integration for motor control. Frontiers in Computational Neuroscience, 8: 1. DOI:10.3389/fncom.2014.00001.

130. Tecchio, F., Salustri, C., Thaut, M. H., Pasqualetti, P., \& Rossini, P. (2000). Conscious and preconscious adaptation to rhythmic auditory stimuli: a magnetoencephalographic study of human brain responses. Experimental Brain Research, 135(2), 222-230.

131. Thaut, M. H. (2003). Neural basis of rhythmic timing networks in the human brain. Annals of the New York Academy of Sciences, 999(1), 364-373.

132. Thaut, M. H. (2005). Rhythm, music, and the brain: Scientific foundations and clinical applications (Vol. 7): Routledge.

133. Thaut, M. H., Gardiner, J. C., Holmberg, D., Horwitz, J., Kent, L., Andrews, G., et al., (2009). Neurologic music therapy improves executive function and emotional adjustment in traumatic brain injury rehabilitation. Annals of the New York Academy of Sciences, 1169(1), 406-416.

134. Thaut, M. H., Leins, A. K., Rice, R. R., Argstatter, H., Kenyon, G. P., McIntosh, G. C., . . . Fetter, M. (2007). Rhythmic auditory stimulation improves gait more than NDT/Bobath training in near-ambulatory patients early poststroke: a single-blind, randomized trial. Neurorehabilotation and Neural Repair, 21(5), 455-459.

135. Thaut, M., Schleiffers, S., \& Davis, W. (1991). Analysis of EMG activity in biceps and triceps muscle in an upper extremity gross motor task under the influence of auditory rhythm. Journal of Music Therapy, 28(2), 64-88.

136. Tierney, A., \& Kraus, N. (2013). The ability to move to a beat is linked to the consistency of neural responses to sound. Journal of Neuroscience, 33(38), 14981-14988.

137. Torres, E. B., Heilman, K. M., \& Poizner, H. (2011). Impaired endogenously evoked automated reaching in Parkinson's disease. Journal of Neuroscience, 31(49), 17848-17863.

138. Vesper, C., Butterfill, S., Knoblich, G., \& Sebanz, N. (2010). A minimal architecture for joint action. Neural Networks, 23(8), 998-1003. 
139. Weeks, D. L., \& Kordus, R. N. (1998). Relative frequency of knowledge of performance and motor skill learning. Research Quarterly for Exercise and Sport, 69(3), 224-230.

140. Welch, R. B. (1978). Perceptual modification. Adapting to Altered Sensory Environments. Academic Press.

141. Whitall, J., Waller, S. M., Sorkin, J. D., Forrester, L. W., Macko, R. F., Hanley, D. F., et al., (2011). Bilateral and unilateral arm training improve motor function through differing neuroplastic mechanisms a single-blinded randomized controlled trial. Neurorehabilitation and Neural Repair, 25(2), 118-129.

142. Wigmore, V., Tong, C., \& Flanagan, J. R. (2002). Visuomotor rotations of varying size and direction compete for single internal model in working memory. Journal of Experimental Psychology: Human Perception and Performance, 28, 447-457.

143. Winstein, C. J. (1991). Knowledge of results and motor learning-implications for physical therapy. Physical therapy, 71(2), 140-149.

144. Wolpert, D. M., \& Kawato, M. (1998). Multiple paired forward and inverse models for motor control. Neural Networks, 11(7-8), 1317-1329.

145. Wolpert, D. M., Diedrichsen, J., \& Flanagan, J. R. (2011). Principles of sensorimotor learning. Nature Reviews Neuroscience, 12, 739-751.

146. Wolpert, D. M., Ghahramani, Z., \& Jordan, M. I. (1995). An internal model for sensorimotor integration. Science, 269, 1880-1882.

147. Young, W. R., Rodger, M. W., \& Craig, C. M. (2014). Auditory observation of stepping actions can cue both spatial and temporal components of gait in Parkinson' s disease patients. Neuropsychologia, 57, 140-153.

148. Zmigrod, S., \& Hommel, B. (2013). Feature Integration across Multimodal Perception and Action: A Review. Multisensory Research 26: 143-157. 